\title{
ADAPTABILIDADE E ESTABILIDADE DE GENÓTIPOS DE MILHO EM DIFERENTES CONDIÇÕES AMBIENTAIS ${ }^{1}$
}

\author{
PEDRO HÉLIO ESTEVAM RIBEIRO², MAGNO ANTONIO PATTO RAMALHO ${ }^{3}$ e DANIEL FURTADO FERREIRA ${ }^{4}$
}

\begin{abstract}
RESUMO - O objetivo deste trabalho foi estudar os fatores de adaptabilidade e estabilidade de 20 genótipos de milho (Zea mays L.), divididos em quatro grupos genéticos (híbridos simples, duplos e triplos e cultivares de polinização livre), em diferentes níveis de fertilidade, épocas de semeadura e locais do Estado de Minas Gerais. Entre os genótipos avaliadas, o que apresentou maior adaptabilidade foi o híbrido triplo Hatã 3012. Observou-se que a produtividade média dos híbridos triplos foi superior à dos demais grupos. Entre os fatores ambientais estudados, verificou-se que ausência de adubação e semeaduras tardias foram os que mais contribuíram para a diminuição do índice ambiental. As diferentes metodologias usadas para estimar os parâmetros de adaptabilidade e estabilidade, apesar de apresentarem resultados semelhantes, forneceram respostas diferenciadas com relação à inclinação das retas de regressão de alguns genótipos. O nível de fertilizantes e a época de semeadura foram os dois fatores de maior influência na determinação da qualidade dos ambientes.
\end{abstract}

Termos para indexação: Zea mays, fatores ambientais, interação genótipo x ambiente, espiga, peso.

\section{ADAPTABILITY AND STABILITY OF CORN GENOTYPES UNDER DIFFERENT ENVIRONMENTAL CONDITIONS}

\begin{abstract}
The objective of this work was to study the adaptability and stability factors of 20 corn genotypes (Zea mays L.) split into four genetical groups (single cross, three way crosses, double crosses and open cultivars) at different fertility levels, sowing dates and sites in the state of Minas Gerais. Among the cultivars evaluated, the three way crosses Hatã 3012 presented greatest adaptability. It was found that the average yield of the three way crosses was superior to those of the other groups, being most adapted. Among environmental factors studied, the absence of fertilization and late sowing were the ones which contributed the most to the decrease of environmental index. The methodologies used to estimate the adaptability and stability parameters, despite showing similar results, provided distinctive responses relative to the inclination of the regression straight line of some genotypes. The level of fertilizers and the sowing date were the two factors of greatest influence in determining the quality of environments.
\end{abstract}

Index terms: Zea mays, environmental factors, genotype $\mathrm{x}$ environment interaction, spikes, weight.

\section{INTRODUÇÃO}

No Brasil existem inúmeras instituições públicas e privadas na área de melhoramento do milho.

\footnotetext{
${ }^{1}$ Aceito para publicação em 11 de janeiro de 2000.

${ }^{2}$ Eng. Agrôn., Dr., Embrapa-Centro de Pesquisa Agroflorestal de Roraima, Caixa Postal 133, CEP 69301-970 Boa Vista, RR. E-mail: pestevam@cpafrr.embrapa.br, pestevan@ufla.br

${ }^{3}$ Eng. Agrôn., Dr., Dep. de Biologia, Universidade Federal de Lavras (UFLA), Caixa Postal 37, CEP 37200-000 Lavras, MG. E-mail: magnoapr@ufla.br

${ }^{4}$ Eng. Agrôn., Dr., Dep. de Estatística, UFLA.

E-mail: danielff@ufla.br
}

O objetivo maior dessas instituições é desenvolver e recomendar genótipos que associem elevado potencial para a produtividade a características agronômicas desejáveis. Todavia, um dos grandes problemas que se tem enfrentado é que, quando os genótipos são postos a competir em vários ambientes, a classificação relativa entre eles pode não ser coincidente, o que dificulta de forma substancial a identificação daqueles efetivamente superiores. A essa oscilação no comportamento dos genótipos, frente às variações ambientais, dá-se o nome de interação genótipos por ambientes. Vários são os relatos que destacam a importância da interação genótipos por ambientes em trabalhos realizados com a cultura do milho no Bra- 
sil (Souza, 1989; Carvalho et al., 1992; Arias, 1995: Gonçalves, 1997).

Para se constatar a presença e também estimar a magnitude da interação é necessário avaliar os genótipos em vários ambientes. Contudo, é possível reduzir os custos e o tempo de experimentação simulando variações ambientais por meio de níveis de fertilizantes e épocas de semeaduras além de outros fatores que podem ser controlados.

Uma vez detectada a interação, há alternativas para se atenuar os seus efeitos. Entre essas alternativas, a mais empregada é a identificação de genótipos com maior estabilidade. Entre as diversas metodologias, as que empregam regressão linear são as mais utilizadas. Inicialmente, a proposta foi de se utilizar apenas um segmento de reta (Finlay \& Wilkinson, 1963; Eberhart \& Russell, 1966). Contudo, foi levantada a hipótese de se identificar genótipos com performance desejável nos ambientes considerados desfavoráveis e favoráveis. Para isso é necessário o emprego de dois segmentos de reta, Verma et al. (1978), método que utiliza dupla análise de regressão linear, em que em cada uma utiliza-se um modelo semelhante ao de Finlay \& Wilkinson (1963) e Eberhart \& Russell (1966). Cruz \& Regazzi (1994) comentam que Silva \& Barreto (1985) propuseram uma análise alternativa, na qual os parâmetros de estabilidade e adaptabilidade fossem estimados por meio do ajuste de uma única equação, representada por uma reta bissegmentada. Cruz et al. (1989) apresentaram uma extensão da metodologia proposta por Silva \& Barreto (1985), tornando-a operacionalmente mais simples e com propriedades estatísticas mais adequadas aos propósitos do melhoramento. Toler (1990) propôs o emprego de um novo modelo de análise de regressão bissegmentado, em que o índice ambiental é um parâmetro a ser estimado juntamente com os $\alpha$ 's e $\beta$ 's. Essa metodologia foi pouco utilizada e seria importante verificar se ela possibilita classificar com eficiência os genótipos com relação à estabilidade nas condições tropicais.

Um método proposto ainda para se avaliar o desempenho de genótipos, em diferentes ambientes, foi apresentado por Annicchiarico (1992). Neste caso, estima-se um índice denominado de índice de confiança. Este índice, tem como característica o fato de possibilitar a recomendação de uma cultivar consi- derando o risco de esta apresentar desempenho abaixo de um dado padrão, como por exemplo, a média geral. A probabilidade de insucesso será tanto menor quanto maior for o índice de confiança.

O presente trabalho teve por objetivo estudar a adaptabilidade e estabilidade de diferentes tipos de genótipos de milho, em 36 ambientes.

\section{MATERIAL E MÉTODOS}

Foram avaliados 20 genótipos de milho, sendo cinco de cada um dos quatro grupos: híbridos simples, triplo e duplos e variedades. Os ensaios foram instalados em três épocas de semeaduras, em três municípios do Estado de Minas Gerais: Lavras (latitude $20^{\circ} 14^{\prime} \mathrm{S}$, longitude $45^{\circ} 0 \mathrm{~W}$, altitude $910 \mathrm{~m}$ ), Lambari (latitude $21^{\circ} 58^{\prime} \mathrm{S}$, longitude $45^{\circ} 22^{\prime} \mathrm{W}$, altitude $845 \mathrm{~m}$ ), Patos de Minas (latitude $18^{\circ} 35^{\prime} \mathrm{S}$, longitude $46^{\circ} 31^{\prime} \mathrm{W}$, altitude $944 \mathrm{~m}$ ) e com quatro níveis de fertilizante (Tabela 1), perfazendo um total de 36 ambientes. As características químicas e físicas dos solos dos locais onde os ensaios foram conduzidos são apresentados na Tabela 2. Em cada local foram conduzidos 12 experimentos distintos, em áreas contíguas, resultantes da combinação de quatro níveis de fertilidade e três épocas de semeadura. Em todos os locais, a primeira época de semeadura deu-se no início da segunda quinzena de outubro; a segunda e terceira épocas, 30 e 60 dias após a primeira, respectivamente. $\mathrm{O}$ delineamento experimental utilizado foi o de blocos casualizados, com três repetições, com parcelas constituídas de duas linhas de quatro metros de comprimento, espaçadas de $0,90 \mathrm{~m}$ e $0,20 \mathrm{~m}$ entre plantas.

O preparo do solo foi semelhante para cada experimento; em todos eles a aração e gradagem foi realizada um a dois dias antes da semeadura. Após a abertura dos sulcos, com cultivador, era realizada a adubação manualmente por linha da parcela. A adubação nitrogenada de cobertura foi realizada aos 35 dias após a emergência utilizando sulfato de amônia (Tabela 1).

A análise de variância conjunta, considerando o peso de espigas despalhadas, foi realizada com os dados corrigidos para umidade de $15 \%$, e para o estande constante de 40 plantas por parcela, por meio da análise de covariância (Vencovsky \& Barriga, 1992).

A partir dos dados médios das análises de variância, foram estimados os parâmetros que avaliam a adaptabilidade e estabilidade utilizando-se os métodos apresentados a seguir:

1) Cruz et al. (1989) a partir do modelo:

$\mathrm{Y}_{\mathrm{ij}}=\beta_{0 \mathrm{i}}+\beta_{1 \mathrm{i}} \mathrm{I}_{\mathrm{j}}+\beta_{2 \mathrm{i}} \mathrm{T}\left(\mathrm{I}_{\mathrm{j}}\right)+\delta_{\mathrm{ij}}+\varepsilon_{\mathrm{ij}}$, 
em que $\mathrm{Y}_{\mathrm{ij}}$ : valor observado do genótipo $\mathrm{i}$, no ambiente $\mathrm{j}$ e na repetição $\mathrm{k} ; \beta_{0 \mathrm{i}}$ : média geral do genótipo $\mathrm{i}$; $\beta_{1 i}$ : coeficiente de regressão linear, avalia a resposta do genótipo i nos ambientes desfavoráveis; $\beta_{2 \mathrm{i}}$ : coeficiente de regressão linear que somado ao $\beta_{1 \mathrm{i}}$ representa a resposta do genótipo i nos ambientes favoráveis; $\mathrm{I}_{\mathrm{j}}$ : índice ambiental (diferença entre a média de cada ambiente e a média geral); $\mathrm{T}\left(\mathrm{I}_{\mathrm{j}}\right)=0$ se $\mathrm{I}_{\mathrm{j}}<0 ; \mathrm{T}\left(\mathrm{I}_{\mathrm{j}}\right)=\mathrm{I}_{\mathrm{j}}-\mathrm{I}_{+}$se $\mathrm{I}_{\mathrm{j}}>0$, sendo $\mathrm{I}_{+}$a média dos índices $\mathrm{I}_{\mathrm{j}}$ positivos; $\delta_{\mathrm{ij}}$ : desvio da regressão do genótipo i no ambiente $\mathrm{j} ; \varepsilon_{\mathrm{ij}}$ : erro médio associado à média.

2) Toler (1990) empregando-se o seguinte modelo:

$Y_{i j}=\alpha_{i}+\left[\beta_{1 i} Z_{j}+\beta^{\circledR}{ }_{2 i}\left(1-Z_{j}\right)\right] \mu_{j}$

em que $\mathrm{Y}_{\mathrm{ij}}$ : resposta média do genótipo $\mathrm{i}$ ao ambiente $\mathrm{j}$;

$\alpha_{\mathrm{i}}$ : valor do intercepto, com $\mu=0 ; \beta_{1 \mathrm{i}}$ e $\beta^{\circledR}{ }_{2 \mathrm{i}}$ : sensividade de resposta do genótipo i, em ambientes desfavorável e favorável, respectivamente, tendo o $\beta^{\circledR}{ }_{2 \mathrm{i}}$ como equivalência no método de Cruz et al. (1989) o $\beta_{1 \mathrm{i}}+\beta_{2 \mathrm{i}}$; $\mu_{\mathrm{j}}$ : referese ao índice ambiental com $Z_{j}=1$ se $\mu_{j} \leq 0$ e $Z_{j}=0$ se $\mu_{j}>0$. Vale ressaltar que nos casos em que o modelo bissegmentado for não significativo, ou seja, rejeita-se a hipótese $\mathrm{H}_{0}: \beta_{1 \mathrm{i}}=\beta^{\circledR}{ }_{2 \mathrm{i}}$, as inferências são feitas com base no modelo linear, análogo ao de Eberhart \& Russell (1966). Toler (1990) sugeriu um critério para caracterizar a resposta dos genótipos frente às variações ambientais. Segundo esse autor, os genótipos podem ser agrupados em cinco categorias de acordo com a significância de $\left(\beta^{\circledR}{ }_{2 \mathrm{i}}-\beta_{1 \mathrm{i}}\right), \beta_{1 \mathrm{i}} \mathrm{e}$ $\beta_{i}$. Por essa classificação, tem-se os seguintes agrupamentos: A) Rejeitar $\mathrm{H}_{0}:\left(\beta_{1 \mathrm{i}}=\beta^{\circledR}{ }_{2 \mathrm{i}}\right)$ e $\beta_{1 \mathrm{i}}<1<\beta^{\circledR}{ }_{2 \mathrm{i}}-$ resposta convexa e duplo desejável; B) Aceitar $\mathrm{H}_{0}$ : $\left(\beta_{1 \mathrm{i}}=\beta^{\circledR}{ }_{2 \mathrm{i}}\right)$ e

TABELA 1. Quantidades de fertilizantes (kg/ha) aplicadas no plantio e em cobertura nos experimentos de genótipos de milho. Ano agrícola 1996/97.

\begin{tabular}{|c|c|c|c|c|c|}
\hline \multirow{2}{*}{$\begin{array}{l}\text { Nível de } \\
\text { fertilizante }\end{array}$} & \multicolumn{2}{|c|}{ Nitrogênio } & \multirow{2}{*}{$\begin{array}{c}\text { Fósforo } \\
\left(\mathrm{P}_{2} \mathrm{O}_{5}\right)\end{array}$} & \multirow{2}{*}{$\begin{array}{c}\text { Potássio } \\
\left(\mathrm{K}_{2} \mathrm{O}\right)\end{array}$} & \multirow[t]{2}{*}{ Zinco } \\
\hline & Plantio & Cobertura & & & \\
\hline Nível 1 & 0 & 0 & 0 & 0 & 0,0 \\
\hline Nível 2 & 14 & 26 & 49 & 28 & 1,4 \\
\hline Nível 3 & 28 & 52 & 98 & 56 & 2,8 \\
\hline Nível 4 & 42 & 78 & 147 & 84 & 4,2 \\
\hline
\end{tabular}

TABELA 2. Características químicas e físicas dos solos nos locais onde os ensaios foram conduzidos.

\begin{tabular}{lccc}
\hline Característica & \multicolumn{3}{c}{ Locais } \\
\cline { 2 - 4 } & Lavras & Lambari & Patos de Minas \\
\hline $\mathrm{pH} \mathrm{em} \mathrm{água}$ & 5,9 & 5,3 & 5,7 \\
$\mathrm{P}\left(\mathrm{mg} / \mathrm{dm}^{3}\right)$ & 9,6 & 15,6 & 38,3 \\
$\mathrm{~K}\left(\mathrm{mmol}_{\mathrm{c}} / \mathrm{dm}^{3}\right)$ & 1,4 & 1,4 & 1,2 \\
$\mathrm{Ca}\left(\mathrm{mmol}_{\mathrm{c}} / \mathrm{dm}^{3}\right)$ & 28,0 & 17,0 & 23,0 \\
$\mathrm{Mg}\left(\mathrm{mmol}_{\mathrm{c}} / \mathrm{dm}^{3}\right)$ & 5,7 & 8,7 & 11,0 \\
Boro $\left(\mathrm{mg} / \mathrm{dm}^{3}\right)$ & 0,3 & 0,2 & 0,3 \\
Zinco $\left(\mathrm{mg}^{3} / \mathrm{dm}^{3}\right)$ & 2,4 & 1,2 & 3,4 \\
Cobre $\left(\mathrm{mg}^{3} \mathrm{dm}^{3}\right)$ & 2,3 & 3,8 & 7,6 \\
$\mathrm{Al}\left(\mathrm{mmol} / \mathrm{dm}^{3}\right)$ & 0,0 & 0,7 & 0,0 \\
$\mathrm{H}+\mathrm{Al}\left(\mathrm{mmol}_{\mathrm{c}} / \mathrm{dm}^{3}\right)$ & 32,3 & 60,7 & 61,0 \\
$\mathrm{~S}\left(\mathrm{mmol} / \mathrm{dm}^{3}\right)$ & 35,1 & 27,7 & 35,2 \\
$\mathrm{CTC}$ efetiva $\left(\mathrm{mmol}_{\mathrm{c}} / \mathrm{dm}^{3}\right)$ & 35,1 & 27,7 & 35,2 \\
$\mathrm{CTC}$ a pH $7\left(\mathrm{mmol}_{\mathrm{c}} / \mathrm{dm}^{3}\right)$ & 67,4 & 87,8 & 96,2 \\
Saturação por Al da CTC efetiva $(\%)$ & 0,0 & 2,6 & 0,0 \\
Saturação por base da CTC a pH 7,0 $(\%)$ & 52,0 & 31,5 & 36,5 \\
Carbono $(\mathrm{g} / \mathrm{kg})$ & 14,7 & 17,3 & 19,0 \\
Matéria orgânica $(\mathrm{g} / \mathrm{kg})$ & 25,3 & 30,0 & 32,7 \\
Areia $(\mathrm{g} / \mathrm{kg})$ & 253,3 & 466,7 & 300,0 \\
Limo $(\mathrm{g} / \mathrm{kg})$ & 300,0 & 243,3 & 413,3 \\
Argila $(\mathrm{g} / \mathrm{kg})$ & 446,7 & 290,0 & 253,3 \\
\hline
\end{tabular}


$\beta_{\mathrm{i}}>1$ - resposta linear simples e desejável só em ambientes de alta qualidade; $C)$ Aceitar $\mathrm{H}_{0}:\left(\beta_{1 \mathrm{i}}=\beta^{\circledR}{ }_{2 \mathrm{i}}\right)$, e $\beta_{\mathrm{i}}=1$ - resposta linear simples, não desviando da resposta média; D) Aceitar $H_{0}:\left(\beta_{1 \mathrm{i}}=\beta^{\circledR}{ }_{2 \mathrm{i}}\right)$ e $\beta_{\mathrm{i}}<1$ - resposta linear simples e desejável só em ambientes de baixa qualidade e E) Rejeitar $\mathrm{H}_{0}:\left(\beta_{1 \mathrm{i}}=\beta^{\circledR}{ }_{2 \mathrm{i}}\right)$ e $\beta_{1 \mathrm{i}}>1>\beta^{\circledR}{ }_{2 \mathrm{i}}-$ resposta côncava e duplo indesejável.

3) Annicchiarico (1992), realizado para estimar o risco de adoção de uma cultivar utilizando-se os dados médios por experimento.

\section{RESULTADOS E DISCUSSÃO}

O milho no Estado de Minas Gerais é cultivado em diferentes condições ambientais, que envolvem ampla variação na fertilidade do solo, na quantidade de fertilizantes utilizada e na época de semeadura. Na recomendação de cultivares, deve-se considerar todas essas condições. O procedimento adotado permitiu estimar os parâmetros de adaptabilidade e estabilidade ao menor custo, porque a parte mais onerosa dos experimentos de avaliação de genótipos é o custo do transporte da equipe e do material para instalação dos experimentos, além do tempo despendido pelo pesquisador durante a locomoção. A simulação de variações ambientais em um ou poucos locais, além de reduzir os custos, promove também maior eficiência experimental, já que esses experimentos podem ser conduzidos em locais com melhor infraestrutura.

Constata-se que, praticamente, todas as fontes de variação apresentaram teste $F$ significativo $(P \leq 0,05)$, exceto as interações cultivar dentro de grupo x níveis, grupo x locais e interações de graus mais elevados, triplas e quádruplas (Tabela 3 ).

Os efeitos de épocas de semeadura e níveis de adubação, no desempenho de genótipos de milho, já foram temas de outros artigos e, portanto, esses efeitos não serão mais discutidos aqui isoladamente, sendo considerados então indistintamente como efeitos ambientais.

Na metodologia de Toler (1990), a estimativa $\mu_{j}$ permite avaliar o que se denomina de qualidade ambiental (Tabela 4). Entre os fatores de variação ambiental, ou seja, níveis de adubação, épocas de semeadura e locais, verificou-se que o nível 1, sem fertilizantes, foi o que mais contribuiu para diminuição da média, com estimativas de $\mu_{\mathrm{j}}$ negativas em todas as épocas e locais. A terceira época foi o segundo fator determinante da baixa qualidade ambiental, uma vez que se observou que, dos 12 experimentos instalados nessa época, apenas os de Lambari apresentaram estimativas de $\mu_{\mathrm{j}}$ positivas naqueles ambientes onde foi utilizada adubação. Observa-se que a primeira época de semeadura, à

TABELA 3. Análise de variância conjunta para peso de espigas despalhadas (kg/ha) de genótipos de milho, em quatro níveis de adubação, três épocas de semeaduras, em três municípios do Estado de Minas Gerais. Ano agrícola 1996/97.

\begin{tabular}{|c|c|c|}
\hline Fonte de variação & GL & Quadrado médio \\
\hline Cultivar (C) & 19 & $58.137 .265,8743 * *$ \\
\hline Grupo (G) & 3 & $146.044 .556,7034 * *$ \\
\hline Cultivar dentro de grupo $(\mathrm{C}(\mathrm{G}))$ & 16 & $41.654 .648,8439 * *$ \\
\hline Nível (N) & 3 & 833.007.085,2940** \\
\hline Época (E) & 2 & $539.792 .869,6104 * *$ \\
\hline Local (L) & 2 & $36.195 .534,0848 * *$ \\
\hline Cultivar $\mathrm{x}$ ambiente & 665 & $1.818 .140,4100 * *$ \\
\hline $\mathrm{C} \times \mathrm{N}$ & 57 & $1.988 .627,1275^{*}$ \\
\hline $\mathrm{G} \times \mathrm{N}$ & 9 & $3.233 .456,6580 *$ \\
\hline $\mathrm{C}(\mathrm{G}) \times \mathrm{N}$ & 48 & $1.755 .221,5906^{\mathrm{ns}}$ \\
\hline $\mathrm{C} \times \mathrm{E}$ & 38 & $3.001 .974,2129 * *$ \\
\hline $\mathrm{G} \times \mathrm{E}$ & 6 & $3.051 .135,5908 *$ \\
\hline $\mathrm{C}(\mathrm{G}) \times \mathrm{E}$ & 32 & $2.992 .756,4546^{* *}$ \\
\hline $\mathrm{C} \times \mathrm{L}$ & 38 & $4.734 .714,7825 * *$ \\
\hline Gx L & 6 & $2.444 .839,6367^{\mathrm{ns}}$ \\
\hline $\mathrm{C}(\mathrm{G}) \times \mathrm{L}$ & 32 & $5.164 .066,3724 * *$ \\
\hline $\mathrm{N} \times \mathrm{E}$ & 6 & $26.700 .597,2328 * *$ \\
\hline $\mathrm{N} \times \mathrm{L}$ & 6 & $36.048 .125,1159 * *$ \\
\hline Ex L & 4 & $79.076 .813,3362 * *$ \\
\hline $\mathrm{C} \times \mathrm{N} \times \mathrm{E}$ & 114 & $1.364 .342,3805^{\mathrm{ns}}$ \\
\hline $\mathrm{G} \times \mathrm{N} \times \mathrm{E}$ & 18 & $883.921,1862^{\mathrm{ns}}$ \\
\hline $\mathrm{C}(\mathrm{G}) \times \mathrm{N} \times \mathrm{E}$ & 96 & $1.454 .421,3545^{\mathrm{ns}}$ \\
\hline $\mathrm{C} \times \mathrm{N} \times \mathrm{L}$ & 114 & $1.619 .690,2924^{\mathrm{ns}}$ \\
\hline Gx N x L & 18 & $1.541 .633,9100^{\mathrm{ns}}$ \\
\hline $\mathrm{C}(\mathrm{G}) \times \mathrm{N} \times \mathrm{L}$ & 96 & $1.634 .325,8641^{\mathrm{ns}}$ \\
\hline $\mathrm{C} \times \mathrm{E} \times \mathrm{L}$ & 76 & $2.322 .138,5359 * *$ \\
\hline Gx Ex L & 12 & $2.436 .890,0440 *$ \\
\hline$C(G) \times E \times L$ & 64 & $2.300 .622,6281 * *$ \\
\hline NxEx L & 12 & $21.234 .179,1965 * *$ \\
\hline $\mathrm{C} \times \mathrm{N} \times \mathrm{E} \times \mathrm{L}$ & 228 & $1.250 .242,0662^{\mathrm{ns}}$ \\
\hline Gx N x Ex L & 36 & $1.343 .990,8475^{\mathrm{ns}}$ \\
\hline $\mathrm{C}(\mathrm{G}) \times \mathrm{N} \times \mathrm{E} \times \mathrm{L}$ & 192 & $1.232 .664,1697^{\mathrm{ns}}$ \\
\hline Repetição (N x E x L) & 72 & $3.490 .377,0700 * *$ \\
\hline Erro & 1368 & $1.366 .265,7500$ \\
\hline Média geral & & $7.789,1421$ \\
\hline $\mathrm{R}^{2}$ & & 0,7930 \\
\hline $\mathrm{CV}(\%)$ & & 15,0064 \\
\hline
\end{tabular}


exceção do nível zero de adubação, apresentou estimativas de $\mu_{\mathrm{j}}$ positivas para todos os demais níveis, o que reforça ser essa época a mais recomendada para o cultivo do milho na região, conforme se tem verificado em trabalhos já realizados anteriormente (Souza, 1989).

TABELA 4. Produtividade média (M) por ambiente de espigas despalhadas $(\mathrm{kg} / \mathrm{ha})$, estimativas do índice ambiental $\left(\mu_{\mathrm{j}}\right)$ e respectivo erro padrão (EP), segundo metodologia de Toler (1990), em genótipos de milho no Estado de Minas Gerais. Ano agrícola 1996/971.

\begin{tabular}{rrrrrrc}
\hline $\mathrm{A}$ & $\mathrm{N}$ & $\mathrm{E}$ & $\mathrm{L}$ & $\mathrm{M}$ & \multicolumn{1}{c}{$\mu_{\mathrm{j}}$} & \multicolumn{1}{c}{$\mathrm{EP}$} \\
\hline 1 & 1 & 1 & 1 & 6.268 & $-1.513,18$ & 146,81 \\
2 & 1 & 1 & 2 & 6.329 & $-1.459,70$ & 146,80 \\
3 & 1 & 1 & 3 & 6.310 & $-1.465,36$ & 146,80 \\
4 & 1 & 2 & 1 & 7.037 & $-815,91$ & 146,81 \\
5 & 1 & 2 & 2 & 6.026 & $-1.793,27$ & 146,84 \\
6 & 1 & 2 & 3 & 6.529 & $-1.311,44$ & 146,80 \\
7 & 1 & 3 & 1 & 5.932 & $-1.839,71$ & 146,84 \\
8 & 1 & 3 & 2 & 4.865 & $-2.946,13$ & 147,13 \\
9 & 1 & 3 & 3 & 4.650 & $-3.131,89$ & 147,21 \\
10 & 2 & 1 & 1 & 9.061 & $1.293,20$ & 145,77 \\
11 & 2 & 1 & 2 & 9.875 & $2.140,09$ & 145,97 \\
12 & 2 & 1 & 3 & 7.856 & 138,71 & 145,93 \\
13 & 2 & 2 & 1 & 8.317 & 480,39 & 145,83 \\
14 & 2 & 2 & 2 & 7.441 & $-318,95$ & 146,88 \\
15 & 2 & 2 & 3 & 8.283 & 476,30 & 145,83 \\
16 & 2 & 3 & 1 & 6.493 & $-1.288,46$ & 146,80 \\
17 & 2 & 3 & 2 & 7.890 & 94,98 & 145,94 \\
18 & 2 & 3 & 3 & 6.852 & $-893,00$ & 146,81 \\
19 & 3 & 1 & 1 & 8.715 & 947,67 & 145,77 \\
20 & 3 & 1 & 2 & 9.849 & $2.074,35$ & 145,95 \\
21 & 3 & 1 & 3 & 9.267 & $1.436,30$ & 145,79 \\
22 & 3 & 2 & 1 & 8.089 & 274,84 & 145,88 \\
23 & 3 & 2 & 2 & 7.608 & $-196,46$ & 146,90 \\
24 & 3 & 2 & 3 & 8.790 & 969,29 & 145,76 \\
25 & 3 & 3 & 1 & 6.674 & $-1.086,18$ & 146,80 \\
26 & 3 & 3 & 2 & 8.516 & 749,56 & 145,78 \\
27 & 3 & 3 & 3 & 7.546 & $-208,50$ & 146,90 \\
28 & 4 & 1 & 1 & 9.333 & $1.551,58$ & 145,81 \\
29 & 4 & 1 & 2 & 10.245 & $2.492,74$ & 146,14 \\
30 & 4 & 1 & 3 & 10.624 & $2.792,77$ & 146,31 \\
31 & 4 & 2 & 1 & 8.919 & $1.089,11$ & 145,76 \\
32 & 4 & 2 & 2 & 8.260 & 493,41 & 145,83 \\
33 & 4 & 2 & 3 & 8.426 & 599,27 & 145,81 \\
34 & 4 & 3 & 1 & 6.378 & $-1.412,56$ & 146,80 \\
35 & 4 & 3 & 2 & 9.546 & $1.714,30$ & 145,84 \\
36 & 4 & 3 & 3 & 7.612 & $-128,16$ & 145,92 \\
\hline & & & & &
\end{tabular}

${ }^{1}$ A: ambientes; N: níveis; E: épocas de semeadura; L: locais.
Em princípio, quando se estuda a interação genótipos x ambientes, o primeiro problema é o conceito de adaptabilidade. Na literatura há uma ampla variação na aplicação desse conceito (Mariotti et al., 1976; Vencovsky \& Barriga, 1992; Cruz \& Regazzi, 1994). Neste trabalho foi adotado o conceito de adaptabilidade definido por Mariotti et al. (1976), em que a adaptabilidade expressa a capacidade do genótipo em assimilar vantajosamente os estímulos ambientais.

Na metodologia de Cruz et al. (1989), a adaptação é avaliada pelas estimativas de $\beta_{0 \mathrm{i}}$, e na metodologia de Toler (1990), pela estimativa de $\alpha_{i}$. Na metodologia de Toler (1990), $\alpha_{i}$ é estimado e corresponde ao intercepto, isto é, ao desempenho da cultivar i quando $\mu_{\mathrm{i}}=0$; na metodologia de Cruz et al. (1989), $\beta_{0 \mathrm{i}}$ corresponde à média geral da cultivar $\mathrm{i}$ nos $\mathrm{n}$ ambientes.

Constata-se que os valores dos parâmetros de adaptação obtidos pelas duas metodologias são bem semelhantes (Tabelas 5 e 6). A correlação entre esses dois parâmetros foi de $96,66 \%$. Do exposto, podese inferir que a metodologia de Toler (1990) foi eficiente em predizer o desempenho médio dos genótipos. Verifica-se, na Tabela 5, que o genótipo com maior adaptabilidade foi o híbrido Hatã 3012 (9.800 kg/ha). Um segundo grupo envolve os híbridos C-909, AG-5011, C-435, XL-660 e Hatã 1001. O híbrido Hatã 3012 esteve entre os primeiros colocados em $72 \%$ dos experimentos, e a cultivar BR-111, ao contrário, entre os de menor desempenho, em $42 \%$.

O bom desempenho do Hatã 3012 é comprovado, também, pela estimativa do índice de confiabilidade (Annicchiarico, 1992). Observa-se que, mesmo com $75 \%$ de probabilidade, a sua performance será $18 \%$ acima da média dos genótipos. No extremo oposto, a cultivar BR-111, terá desempenho de $24 \%$ abaixo da média, no nível de probabilidade considerado. Pode-se também observar pelo índice de confiabilidade $\left(\mathrm{I}_{\mathrm{i}}\right)$ que as cultivares apresentam desempenho abaixo da média, ou seja, sua recomendação envolve riscos maiores do que a recomendação de híbridos. Tal fato pode ser confirmado quando se analisam os genótipos por grupo, pois se observa que as cultivares irão produzir no máximo $96 \%$ da média dos grupos, e que o grupo dos híbridos triplos produzirá $15 \%$ a mais em relação à média dos demais (Tabela 6). 
Quando se avalia o desempenho médio dos grupos na Tabela 5 , através dos $\beta_{0 \mathrm{i}}$, constata-se que o grupo dos híbridos triplos foi o mais adaptado e o das cultivares o menos adaptado. Nesse contexto, ressalta-se que, em média, o desempenho das cultivares foi $10 \%$ inferior à média dos grupos dos híbridos e $15 \%$ em relação à média dos híbridos triplos. A menor produtividade das cultivares em relação aos híbridos é esperada, haja vista que uma cultivar de milho é composta por um infinito número de híbridos simples, ao passo que os híbridos, sejam simples, triplos ou duplos, são teoricamente as melhores combinações híbridas específicas que podem ser obtidas dentro de uma ou mais cultivares. Resultados que mostram a superioridade dos híbridos em relação às cultivares já foram obtidos em outras opor- tunidades na região (Muniz, 1995). Vale salientar, entretanto, que, dentro do grupo das cultivares, a BR-106 e S. Dentado apresentaram desempenhos estatisticamente iguais e até mesmo superiores aos de alguns híbridos comerciais. Cabe ressaltar que a BR-106 tem apresentado em outras oportunidades bom potencial produtivo e vem sendo utilizada em vários programas de melhoramento para extração de linhagens e obtenção de híbridos (Arias, 1995).

Em estudos da estabilidade dos materiais genéticos, entre os procedimentos mais empregados, estão aqueles que utilizam regressão (Becker \& Léon, 1988; Crossa, 1990; Kang, 1998). Quando se adota regressão, o primeiro questionamento é se apenas um segmento de reta é suficiente para explicar os resultados ou se existe um segmento de reta específico

TABELA 5. Estimativas de parâmetros de adaptabilidade e estabilidade de genótipos de milho segundo o modelo de Cruz et al. (1989), considerando o caráter peso de espigas despalhadas (kg/ha), em 36 ambientes no Estado de Minas Gerais. Ano agrícola 1996/971.

\begin{tabular}{lccccccc}
\hline Genótipo & MD & MF & $\beta_{0 \mathrm{i}}$ & $\beta_{1 \mathrm{i}}$ & $\beta_{2 \mathrm{i}}$ & $\beta_{1 \mathrm{i}}+\beta_{2 \mathrm{i}}$ & $\mathrm{R}^{2}$ \\
\hline C-909 & 7.186 & 9.424 & $8.367 \mathrm{e}$ & 0,9045 & $-0,2410$ & 0,6634 & 82,64 \\
Zeneca-8392 & 6.646 & 8.963 & $7.869 \mathrm{~d}$ & 0,9344 & $-0,4951^{* *}$ & $0,4393^{*}$ & 76,71 \\
AG-9012 & 5.747 & 8.332 & $7.111 \mathrm{~b}$ & 0,9923 & $-0,0445$ & 0,9478 & 73,94 \\
XL-220 & 5.773 & 8.306 & $7.110 \mathrm{~b}$ & 1,0699 & 0,2986 & 1,3685 & 85,50 \\
BR-201 (M) & 6.577 & 9.025 & $7.869 \mathrm{~d}$ & 0,9830 & 0,1929 & 1,1760 & 73,39 \\
\hline H. Simples & 6.386 & 8.810 & $7.665 \mathrm{~B}$ & 0,9768 & $-0,0578$ & 0,9190 & 95,40 \\
\hline C-808 & 6.719 & 9.339 & $8.102 \mathrm{~d}$ & 1,0823 & $-0,2798$ & 0,8026 & 86,04 \\
Hatã-3012 & 8.604 & 10.868 & $9.800 \mathrm{f}$ & 0,9067 & $-0,1390$ & 0,7677 & 74,58 \\
XL-360 & 6.359 & 8.900 & $7.700 \mathrm{c}$ & 1,0490 & $-0,1410$ & 0,9081 & 68,00 \\
AG-5011 & 7.412 & 9.661 & $8.599 \mathrm{e}$ & 0,9392 & $-0,1362$ & 0,8030 & 85,14 \\
BR-3123 & 6.809 & 9.082 & $8.008 \mathrm{~d}$ & 0,9895 & $0,4516^{* *}$ & 1,4411 & 81,65 \\
\hline H. Triplo & 7.180 & 9.570 & $8.442 \mathrm{D}$ & 0,9934 & $-0,0489$ & 0,9445 & 96,28 \\
\hline C-701 & 5.620 & 8.506 & $7.143 \mathrm{~b}$ & $1,2164^{*}$ & $-0,1105$ & 1,1060 & 87,60 \\
C-435 & 6.832 & 9.564 & $8.274 \mathrm{E}$ & 1,0793 & $-0,1379$ & 0,9414 & 85,94 \\
XL-660 & 6.702 & 9.549 & $8.205 \mathrm{E}$ & $1,1766^{* *}$ & 0,2044 & 1,3811 & 89,84 \\
Hatã-1001 & 7.114 & 9.701 & $8.479 \mathrm{E}$ & 0,9455 & 0,0134 & 0,9589 & 77,00 \\
BR-205 & 6.113 & 8.209 & $7.219 \mathrm{~B}$ & 1,0094 & $-0,1427$ & 0,8667 & 79,16 \\
\hline H. Duplo & 6.476 & 9.106 & $7.864 \mathrm{C}$ & 1,0855 & $-0,0347$ & 1,0508 & 97,91 \\
\hline BR-106 & 6.338 & 8.618 & $7.541 \mathrm{C}$ & 0,9770 & 0,2390 & 1,2160 & 81,19 \\
BR-111 & 5.270 & 7.735 & $6.571 \mathrm{~A}$ & 0,9838 & 0,3298 & 1,3136 & 80,95 \\
S. Dentado & 6.518 & 8.457 & $7.541 \mathrm{C}$ & $0,8352^{* *}$ & 0,1778 & 1,0130 & 76,59 \\
S. Duro & 5.752 & 8.173 & $7.030 \mathrm{~B}$ & 0,9742 & $-0,0051$ & 0,9691 & 82,10 \\
CMS-39 & 5.964 & 8.388 & $7.243 \mathrm{~B}$ & 0,9518 & $-0,0349$ & 0,9169 & 78,03 \\
\hline Cultivares & 5.968 & 8.274 & $7.185 \mathrm{~A}$ & 0,9444 & 0,1413 & 1,0857 & 95,94 \\
\hline
\end{tabular}

1 Médias seguidas de mesmas letras não diferem entre si pelo teste de Scott \& Knott (1974) a 5\% de probabilidade, com letras maiúsculas para teste feito entre grupos e letras minúsculas para teste entre genótipos; MD: médias em ambientes desfavoráveis; MF: médias em ambientes favoráveis. * e ** Significativo a $5 \%$ e $1 \%$ de probabilidade, respectivamente, pelo teste $t$. 
para os ambientes favoráveis e outro para os ambientes desfavoráveis (Verma et al., 1978; Toler, 1990). As duas metodologias, que utilizam análises de regressão, consideradas neste trabalho permitem obter essa inferência. No caso do método de Cruz et al. (1989), a rejeição da hipótese que $\beta_{2 \mathrm{i}}=0$ indica que o comportamento dos genótipos, em respostas às mudanças dos ambientes, é bissegmentado. Isso ocorreu apenas no caso dos genótipos Zeneca 8392 e BR-3123 (Tabela 4). Na metodologia de Toler (1990), a hipótese testada é se $\beta^{\circledR}{ }_{2 \mathrm{i}}-\beta_{1 \mathrm{i}}=0$ (Tabela 6). A rejeição dessa hipótese fornece a mesma interpretação comentada no método anterior, isto é, que há necessidade de se mudar a inclinação da reta para explicar o desempenho dos genótipos nos ambientes considerados favoráveis. Nesse caso, o contraste foi significativo nos genótipos BR-205 e Hatã 1001.
Nos demais, a resposta pode ser explicada por apenas um segmento de reta. Constata-se, portanto, que as duas metodologias não forneceram os mesmos resultados no que se refere à inclinação das retas dos genótipos Zeneca 8392, BR-3123, BR-205 e Hatã 1001. Para os demais casos, em princípio, houve coerência. Além disso, constatou-se que, no caso dos híbridos C-701 e XL-660, em ambos os métodos os $\beta$ 's $\left(\beta_{1 i}\right.$ e $\left.\beta_{i}\right)$ foram estatisticamente superiores à unidade, indicando que os dois genótipos são responsivos à melhoria do ambiente, já a cultivar S. Dentado, pela metodologia de Cruz et al. (1989), apresentou $\beta_{1 \mathrm{i}}$ menor que a unidade, sendo portanto um material menos responsivo. Os demais genótipos, cujo comportamento foi explicado por apenas uma reta de regressão, apresentaram estimativas de $\beta_{1}$, não diferindo da unidade em ambos os métodos,

TABELA 6. Estimativas de parâmetros de adaptabilidade e estabilidade para genótipos de milho, segundo o método de Toler (1990) e Annicchiarico (1992), considerando o caráter peso de espigas despalhadas (kg/ha), em 36 ambientes no Estado de Minas Gerais. Ano agrícola 1996/97¹.

\begin{tabular}{lcccccr}
\hline Genótipo & $\alpha_{\mathrm{i}}$ & $\beta_{\mathrm{i}} \mathrm{e} \beta_{1 \mathrm{i}}$ & $\beta^{\circledR}{ }_{2 \mathrm{i}}{ } \beta_{1 \mathrm{i}}$ & $\beta^{\circledR}{ }_{2 \mathrm{i}}$ & $\mathrm{R}^{2}$ & $\mathrm{I}_{\mathrm{i}}$ \\
\hline C-909 & 8.486 & $0,8653^{\mathrm{ns}}$ & $-0,1958^{\mathrm{ns}}$ & - & 90,20 & 102 \\
Zeneca-8392 & 8.136 & $0,8560^{*}$ & $-0,4412^{\text {ns }}$ & - & 85,42 & 94 \\
AG-9012 & 6.969 & $0,9869^{\text {ns }}$ & $0,2348^{\text {ns }}$ & - & 86,09 & 83 \\
XL-220 & 6.997 & $1,1185^{\text {ns }}$ & $0,1861^{\text {ns }}$ & - & 92,29 & 83 \\
BR-201 (M) & 7.668 & $1,0122^{\text {ns }}$ & $0,3315^{\text {ns }}$ & - & 85,44 & 93 \\
\hline H. Simples & 7.655 & $0,9677^{\text {ns }}$ & $0,0171^{\text {ns }}$ & - & 89,06 & 103 \\
\hline C-808 & 8.293 & $1,0391^{\text {ns }}$ & $-0,3160^{\text {ns }}$ & - & 92,27 & 98 \\
Hatã-3012 & 9.828 & $0,8918^{\text {ns }}$ & $-0,0470^{\text {ns }}$ & - & 85,85 & 118 \\
XL-360 & 7.846 & $1,0260^{\text {ns }}$ & $-0,2416^{\text {ns }}$ & - & 82,23 & 89 \\
AG-5011 & 8.730 & $0,9190^{\text {ns }}$ & $-0,2165^{\text {ns }}$ & - & 92,18 & 106 \\
BR-3123 & 7.870 & $1,0596^{\text {ns }}$ & $0,2282^{\text {ns }}$ & - & 89,42 & 96 \\
\hline H. Triplo & 8.508 & $0,9859^{\text {ns }}$ & $-0,1099^{\text {ns }}$ & - & 90,26 & 115 \\
\hline C-701 & 7.297 & $1,2025^{* *}$ & $-0,2547^{\text {ns }}$ & - & 93,77 & 83 \\
C-435 & 8.266 & $1,0586^{\text {ns }}$ & $0,0138^{\text {ns }}$ & - & 92,62 & 100 \\
XL-660 & 8.081 & $1,2095^{* *}$ & $0,2034^{\text {ns }}$ & - & 94,74 & 99 \\
Hatã-1001 & 8.153 & $0,6862^{*}$ & $0,5393^{*}$ & $1,2255^{\text {ns }}$ & 88,60 & 101 \\
BR-205 & 7.718 & $1,3838^{* *}$ & $-0,8239^{* *}$ & $0,5599^{* *}$ & 91,23 & 84 \\
\hline H. Duplo & 7.905 & $1,0808^{* *}$ & $-0,0676^{\text {ns }}$ & - & 92,74 & 104 \\
\hline BR-106 & 7.510 & $1,0147^{\text {ns }}$ & $0,0514^{\text {ns }}$ & - & 89,89 & 90 \\
BR-111 & 6.283 & $1,0355^{\text {ns }}$ & $0,4755^{\text {ns }}$ & - & 89,51 & 76 \\
S. Dentado & 7.530 & $0,8620^{\text {ns }}$ & $0,0187^{\text {ns }}$ & - & 87,24 & 90 \\
S. Duro & 6.983 & $0,9731^{\text {ns }}$ & $0,0769^{\text {ns }}$ & - & 90,58 & 83 \\
CMS-39 & 7.136 & $0,9462^{\text {ns }}$ & $0,1770^{\text {ns }}$ & - & 88,30 & 86 \\
\hline Cultivares & 7.089 & $0,9655^{\text {ns }}$ & $0,1605^{\text {ns }}$ & - & 89,77 & 96 \\
\hline
\end{tabular}

${ }^{1}$ Nos casos em que $\left(\beta^{\circledR}{ }_{2 i}-\beta_{1 i}\right)$ foi não-significativo, as estimativas são referentes ao modelo linear; $\mathrm{I}_{\mathrm{i}}$ : índice de confiabilidade de Annicchiarico (1992). ns, $* \mathrm{e}^{* *}$ Não-significativo e significativo a $5 \%$ e $1 \%$ de probabilidade, respectivamente, pelo teste $\mathrm{t}$. 
portanto considerados como de comportamento paralelo à média do ambiente.

Considerando a classificação de Toler (1990) para caracterizar os genótipos frente às variações ambientais, verifica-se que o híbrido BR-205, que apresentou comportamento bissegmentado (Tabela 6) mostrou resposta que a enquadra na categoria $\mathrm{E}$, isto é, apresentou $\beta_{1 \mathrm{i}}$ maior que 1 e $\beta^{\circledR}{ }_{2 \mathrm{i}}$ menor que 1 . Dizendo de outro modo, sua resposta foi côncava e duplo indesejável, ou seja, foi exigente em ambientes desfavoráveis e não responsivo à melhoria do ambiente. Já Hatã 1001, outra cultivar com resposta bissegmentada, apresentou $\beta_{1 \mathrm{i}}=0,69$ e $\beta^{\circledR}{ }_{2 \mathrm{i}}=1,28$, portanto, enquadra-se na categoria $A$, uma vez que se adapta bem em ambientes desfavoráveis e foi responsiva nos ambientes de alta qualidade, sendo considerada, assim, como duplo desejável (Toler, 1990). Os demais genótipos, cuja resposta foi explicada por apenas um segmento de reta, foram enquadrados nas categorias B, C e D. Os genótipos C-701 e XL-660, cujo $\beta_{\mathrm{i}}$ foi superior à unidade (Tabela 6), enquadram-se na categoria $\mathrm{B}$, apresentam resposta linear simples e são responsivos à melhoria do ambiente. Já a cultivar Zeneca 8392, com $\beta_{i}$ menor que 1 , enquadra-se na categoria $\mathrm{D}$, isto é, não é responsiva à melhoria do ambiente, porém é recomendada para ambientes de baixa qualidade. Os demais genótipos foram enquadrados na categoria $C$, ou seja, apresentaram $\beta_{\mathrm{i}}$, não diferindo estatisticamente da unidade, isto é, acompanhando a resposta média do ambiente.

Com relação aos grupos de genótipos, observouse, por meio dos métodos que utilizam regressão, que todos apresentaram comportamento que pode ser explicado por apenas um segmento de reta. Pela metodologia de Cruz et al. (1989), todos os grupos apresentaram $\beta_{1 i}$ iguais à unidade. Por sua vez, analisando-se a significância dos $\beta_{\text {i pela metodologia }}$ de Toler (1990), verifica-se que o grupo H. Duplo apresentou estimativa de $\beta_{\mathrm{i}}$ maior que a unidade (Tabela 6), com recomendação apenas para ambientes de alta qualidade, e enquadrou-se na categoria B. Os demais grupos apresentaram valores de $\beta_{i}$ iguais a 1 , enquadrando-se na categoria $\mathrm{C}$, ou seja, o desempenho acompanha o desempenho da média do ambiente.

Como se constata, essa classificação simplifica a interpretação dos resultados de estabilidade. Contu- do, ela por si só não possibilita a recomendação dos genótipos, pois não considera a adaptação, isto é, a produtividade média do material. Nesse sentido, Toler (1990) propõe que a avaliação do padrão de resposta ao ambiente seja vinculada com a adaptabilidade.

Quando se avaliam diferentes tipos de genótipos, híbridos simples, triplos e duplos e cultivares, por exemplo, em diferentes condições ambientais, um questionamento que surge é com relação à maior ou menor estabilidade dos materiais em função do grupo a que pertencem. Allard \& Bradshaw (1964) comentaram que o fator estabilidade pode ser analisado considerando-se duas situações: estabilidade populacional (homeostase populacional) e estabilidade individual (homeostase individual). A homeostase populacional pressupõe que cada indivíduo que compõe a população seja adaptado a uma diferente faixa de variação ambiental, ao passo que a homeostase individual é conseqüência de uma reação tamponante de cada indivíduo da população, que se adapta a diversos ambientes. Daí percebe-se que populações de base genética estreita dependem mais da homeostase individual para conservar seus caracteres, e nas populações de base genética ampla, estão presentes os dois tipos de homeostase. Nesse contexto, Becker \& Léon (1988) comentam ainda que todos os tipos de genótipos podem ser caracterizados basicamente sob dois aspectos: o grau de heterozigosidade das plantas e a heterogeneidade genética da cultivar. Os autores comentam ainda que a estrutura genética da população pode influenciar no resultado da interação genótipos $\mathrm{x}$ ambientes, uma vez que é esperado que genótipos heterozigotos sejam menos suscetíveis às variações ambientais que os homozigotos, e que populações heterogêneas sejam mais tolerantes que as homogêneas.

Existem inúmeros trabalhos com a cultura do milho que permitem inferir não haver uma relação fixa quanto à homogeneidade ou heterogeneidade do material e sua estabilidade, pois é possível selecionar materiais mais estáveis em qualquer grupo: cultivares, híbridos duplos, triplos e simples (Lemos, 1976; Naspolini Filho, 1976; Gomes, 1990; Muniz, 1995), o que foi constatado também neste trabalho.

A previsibilidade de comportamento pode ser avaliada pela estimativa de $\mathrm{R}^{2}$ na metodologia de Cruz 
et al. (1989). Constatou-se (Tabela 5) que a menor estimativa para esse parâmetro foi obtida com o híbrido XL-360, sendo, portanto, esse o material de menor previsibilidade. Com relação aos demais genótipos, as diferenças não foram tão acentuadas. Considerando-se, em termos percentuais, que a estimativa do valor de $\mathrm{R}^{2}$ pode variar de 0 a $100 \%$, e com base nos resultados contidos na literatura com a cultura do milho, pode-se inferir que os genótipos avaliados, via de regra, apresentaram considerável nível de estabilidade, uma vez que mais de $50 \%$ tiveram valores de $\mathrm{R}^{2}$ superiores a $80 \%$. Observa-se, ainda, que em cada grupo existe pelo menos dois genótipos com $\mathrm{R}^{2}$ superior a $80 \%$, o que permite inferir que a maior ou menor estabilidade dos genótipos independe do grupo a que pertencem. No caso da metodologia de Toler (1990), a estimativa de $\mathrm{R}^{2}$ apresentada reflete a eficiência do modelo em predizer o desempenho do genótipo i no ambiente j, em relação ao valor de $Y_{i j}$ observado. Verifica-se (Tabela 6) que os valores de $\mathrm{R}^{2}$ foram todos altos, com valores acima de $82 \%$, indicando que o modelo foi eficiente em predizer o desempenho dos genótipos nos diferentes ambientes.

\section{CONCLUSÕES}

1. Os genótipos e os grupos de genótipos diferem quanto à adaptabilidade e estabilidade de produção de espigas despalhadas.

2. Os híbridos triplos são os que apresentam menor risco de baixa produção.

\section{REFERÊNCIAS}

ALLARD, R.W.; BRADSHAW, A.D. Implications of genotypes-environmental interactions in applied plant breeding. Crop Science, Madison, v.4, n.5, p.503-508, Sept./Oct. 1964.

ANNICCHIARICO, P. Cultivar adaptations and recommendation from alfalfa trials in Northern Italy. Journal of Genetics \& Breeding, Rome, v.46, n.1, p.269-278, Mar. 1992.

ARIAS, C.A.A. Componentes de variância e covariância genética relacionados à seleção recorrente intra $e$ interpopulacional no milho (Zea mays $L$.). Piracicaba : ESALQ, 1995. 139p. Tese de Doutorado.
BECKER, H.C.; LÉON, J. Stability analysis in plant breeding. Plant Breeding, Berlin, v.101, n.1, p.123, Apr. 1988.

CARVALHO, H.W.L. de; MAGNAVACA, R.; LEAL, M.L.S. Estabilidade da produção de cultivares de milho no Estado de Sergipe. Pesquisa Agropecuária Brasileira, Brasília, v.27, n.7, p.1073-1082, jul. 1992.

CROSSA, J. Statistical analysis of multilocations trials. Advances in Agronomy, San Diego, v.44, p.55-85, 1990.

CRUZ, C.D.; REGAZZI, A.J. Modelos biométricos aplicados ao melhoramento genético. Viçosa : UFV, 1994. 390p.

CRUZ, C.D.; TORRES, R.A. de A.; VENCOVSKY, R. An alternative approach to the stability analysis proposed by Silva e Barreto. Revista Brasileira de Genética, Ribeirão Preto, v.12, n.2, p.567-580, Apr.June 1989.

EBERHART, S.A.; RUSSELL, W.A. Stability parameters for comparing varieties. Crop Science, Madison, v.6, n.1, p.36-40, Jan./Feb. 1966.

FINLAY, K.W.; WILKINSON, G.N. The analysis of adaptation in a plant-breeding programme. Australian Journal of Agriculture Research, Collingwood, v.14, n.6, p.742-754, Jan. 1963.

GOMES, L.S. Interação genótipos x épocas de plantio em milho (Zea mays, L.) em dois locais do oeste do Paraná. Piracicaba : ESALQ, 1990. 148p. Dissertação de Mestrado.

GONÇALVES, F.M.A. Adaptabilidade e estabilidade de cultivares de milho avaliadas em "safrinha" no período de 1993 a 1995. Lavras : UFLA, 1997. 86p. Dissertação de Mestrado.

KANG, M.S. Using genotype-by-environment interaction for crop cultivar development. Advances in Agronomy, San Diego, v.62, p.199-252,1998.

LEMOS, M.A Variabilidade fenotípica em híbridos simples, híbridos duplos, variedades e compostos de milho. Piracicaba : ESALQ, 1976. 62p. Dissertação de Mestrado.

MARIOTTI, J.A.; OYARZABAL, E.S.; OSA, J.M.; BULACIO, A.N.R.; ALMADA, G.H. Análisis de estabilidad y adaptabilidad de genotipos de cana de azúcar. I. Interacciones dentro de un localidad ex- 
perimental. Revista Agronómica del Noroeste Argentino, San Miguel de Tucumán, v.13, n.14, p.105127, ene. 1976.

MUNIZ, J.A. Avaliação da estabilidade de cultivares de milho em diferentes níveis de adubação e locais da região de Lavras, MG. Lavras : UFLA, 1995. 60p. Dissertação de Mestrado.

NASPOLINI FILHO, V. Variabilidade fenotípica e estabilidade em híbridos simples, híbridos duplos, variedades e compostos de milho. Piracicaba : ESALQ, 1976. 68p. Dissertação de Mestrado.

SCOTT, A.J.; KNOTT, M. A cluster analysis method for grouping means in the analysis of variance. Biometrics, Washington, n.30, p.507-512, 1974.
SOUZA, F.R.S. Estabilidade de cultivares de milho (Zea mays $\mathrm{L}$.) em diferentes épocas e locais de plantio em Minas Gerais. Lavras : ESAL, 1989. 80p. Dissertação de Mestrado.

TOLER, J.E. Patterns of genotypic performance over environmental arrays. Clemson : Clemson University, 1990. 154p. Ph.D. Thesis.

VENCOVSKY, R.; BARRIGA, P. Genética biométrica no fitomelhoramento. Ribeirão Preto : Sociedade Brasileira de Genética, 1992. 496p.

VERMA, M.M.; CHAHAL, G.S.; MURTY, B.R. Limitation of conventional regression analysis: a proposed modification. Theoretical and Applied Genetics, Berlin, v.53, n.1, p.89-91, Jan. 1978. 